\section{Iron therapy and infection}

(MARCH 2011)

TO THE EDITOR: In their article, "Is iron therapy for anemia harmful in the setting of infection?" in the March 2011 issue, Daoud et $\mathrm{al}^{1}$ illustrated an interesting aspect we often encounter, especially in nephrology practice. However, I believe several points should be clarified in this context.

First, "iron therapy" and "iron stores" are quite different things when we talk about infection. As Daoud et al state, human studies involving iron therapy and infection are conflicting in their results. The explanation is likely that iron therapy per se does not always translate to increased iron stores, while iron stores do correlate with increased risk of infection and death, whether in hemodialysis patients ${ }^{2}$ or in the general population. ${ }^{3}$ Intravenous iron therapy mostly gains the association with risk of infection when dosed greater than a certain amount or for an extended duration. In addition, Pieracci et $\mathrm{al}^{4}$ showed that oral iron therapy for anemia does not boost the infection rate during critical illness when equivalent iron markers are achieved.

This mounting evidence solidifies the view that iron stores underlie the infection susceptibility. But to prove this concept, a randomized controlled study consisting of achieving similar iron stores by component therapy or intravenous iron supplementation would be the best option.

Second, I wish to add a category of infection omitted in their article, ie, fungal infection (mucormycosis). Mucormycosis, a rare but life-threatening disease, is caused by fungi of the class Zygomycetes that spread systemically in immunocompromised hosts, with a high death rate. Iron overload, whether or not accompanied by the use of deferoxamine (Desferal), is an established risk factor for mucormycosis. These fungi possess a highaffinity iron permease and produce siderophores, both of which facilitate the uptake of iron. ${ }^{5}$ An abundant host iron pool further enhances their scavenging process, resulting in devastating proliferation and tissue damage. This disease category should be borne in mind when dealing with immunocompromised patients undergoing iron therapy.

\author{
CHIA-TER CHAO, MD \\ National Taiwan University Hospital \\ Taipei, Taiwan
}

REFERENCES

1. Daoud E, Nakhla E, Sharma R. Is iron therapy for anemia harmful in the setting of infection? Cleve Clin J Med 2011; 78:168-170.

2. Pieracci FM, Barie PS. Iron and the risk of infection. Surg Infect 2005; 6(suppl 1):S41-S46.

3. Ellervik C, Tybjærg-Hansen A, Nordestgaard BG. Total mortality by transferrin saturation levels: two general population studies and a metaanalysis. Clin Chem 2011; 57:459-466.

4. Pieracci FM, Henderson P, Rodney JR, et al. Randomized, double-blind, placebo-controlled trial of effects of enteral iron supplementation on anemia and risk of infection during surgical critical illness. Surg Infect 2009; 10:9-19.

5. Ibrahim A, Spellberg B, Edwards J Jr. Iron acquisition: a novel perspective on mucormycosis pathogenesis and treatment. Curr Opin Infect Dis 2008; 21:620-625.

doi:10.3949/ccjm.78c.06001

IN REPLY: We agree that iron therapy is different than iron stores, but iron therapy should be started on the basis of depleted iron stores; otherwise, it is unjustifiable. We also agree that elevated iron stores are dangerous in the setting of infection, more than iron therapy itself. This is really an unproven theory. Most studies that showed worse outcomes of iron therapy found that elevated ferritin is a risk factor. ${ }^{1}$ The problem, as we outlined in our paper, is that most serum markers of iron are unreliable in case of inflammation or infection or in the critically ill. ${ }^{2}$ Evaluation of bone marrow stores is probably the most accurate. $^{3}$

EHAB DAOUD, MD Cleveland Clinic

\section{- REFERENCES}

1. Cavill I. Intravenous iron as adjuvant therapy: a twoedged sword? Nephrol Dial Transplant 2003; 18(suppl 8):viii24-viii28.

2. Pieracci FM, Barie PS. Diagnosis and management of iron-related anemias in critical illness. Crit Care Med 2006; 34:1898-1905.

3. Wish JB. Assessing iron status: beyond serum ferritin and transferrin saturation. Clin J Am Soc Nephrol 2006; 1(suppl 1):S4-S8.

doi:10.3949/ccjm.78c.06002 


\section{Managing bloodstream infections}

(JANUARY 2011)

TO THE EDITOR: I congratulate Drs. O'Grady and Chertow for their excellent review on bloodstream infections. ${ }^{1}$ I just want to call attention to one aspect that the authors forgot. In FIGURE 1, they classified patients as being mildly or moderately ill if they had no hypotension or organ failure, and subdivided this group into those having or not having high-risk factors. The high-risk factors included evidence of severe sepsis, which by definition needs dysfunction or failure of one or more organs. ${ }^{2}$

As has been demonstrated by epidemiologic studies, severe sepsis is associated with a high risk of death, ${ }^{3}$ twice as high as in patients with only catheter-related bloodstream infection. ${ }^{4}$ So, according to the joint guidelines of the American College of Chest Physicians and the Society of Critical Care Medicine, ${ }^{2}$ severe sepsis implies dysfunction or failure of at least one organ. I believe that patients with severe sepsis should be classified in the group of seriously ill.

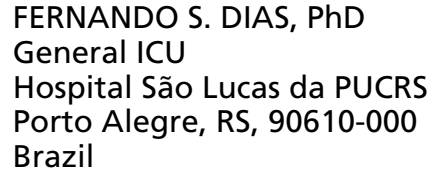

\footnotetext{
$\because$ REFERENCES

1. O'Grady NP, Chertow DS. Managing bloodstream infections in patients who have short-term central venous catheters. Clev Clin J Med 2011; 78:10-17.

2. Bone RC, Balk RA, Cerra FB, et al. Definitions for sepsis and organ failure and guidelines for the use of innovative therapies in sepsis. The ACCP/SCCM Consensus Conference Committee. American College of Chest Physicians/Society of Critical Care Medicine. Chest 1992; 101:1644-1655.

3. Vincent J-L, Sakr Y, Sprung CL et al; Sepsis Occurrence in Acutely III Patients Investigators. Sepsis in European intensive care units: results of the SOAP study. Crit Care Med 2006; 34:344-353.

4. Zias N, Chroneou A, Beamis JF, Craven DE. Vascular catheter-related bloodstream infections. In: O'Donnell JM, Nácul FE, editors. Surgical Intensive Care, 2nd Edition. New York: Springer, 2010:311-324.
}

doi:10.3949/ccjm.78c.06003
IN REPLY: We thank Dr. Dias for his careful read of our article, "Managing bloodstream infections in patients who have short-term central venous catheters," and we acknowledge that he is correct to point out that, by definition, severe sepsis is sepsis associated with organ dysfunction, hypoperfusion, or hypotension. Given this, he is correct that patients with severe sepsis should be categorized in the "seriously ill" patient group in our FIGURE 1.

In effect, however, the recommendations for patients in the "high-risk-factor" group are the same as the recommendations for the "seriously ill" patient group, which are to remove the catheter, draw at least two sets of blood cultures with at least one from a peripheral vein, and start empiric antibiotic therapy.

DANIEL S. CHERTOW, MD National Institutes of Health Bethesda, MD

NAOMI O'GRADY, MD National Institutes of Health Bethesda, MD

doi:10.3949/ccjm.78c.06004 